\title{
Investigating the Gene Expression Profiles of Immune Checkpoints in Breast Cancer Microenvironment and Peripheral Blood: A Comparative Study
}

\author{
Howraa J Bakal ${ }^{1}$, Khairallah A.S Mohammed ${ }^{2}$, Naael H. Ali ${ }^{3}$ \\ \{horaa.j.al@gmail.com ${ }^{1}$,dr.kmohammed@stu.edu.iq ${ }^{2}$,nhali66@yahoo.com ${ }^{3}$ \} \\ Department of Medical Lab Technology, College of Health and Medical Technology, Southern \\ Technical University, Iraq ${ }^{1,2}$ \\ College of Medicine, University of Basra, Iraq $^{1}$
}

\begin{abstract}
The goal of this study was to compare and assess the expression of immunological checkpoints (FOXP3 and CTLA-4) in the tumour microenvironment and peripheral blood in breast cancer patients. The CTLA-4 and FOXP3 transcripts was measured using real-time PCR after total RNA was splitted from blood and tissues from 36 women with breast cancer and 15 women with breast benign tumours. CTLA-4 expression was considerably higher in the tissues of breast cancer patients than in the blood $(\mathrm{P}<0.02)$. CTLA-4 expression was considerably lower in blood from individuals with breast cancer compared to blood from the benign group $(\mathrm{P}<0.001)$. There was no difference in CTLA-4 mRNA expression in tissues and blood in a breast benign tumour. FOXP3 was higher in tissue from breast cancer than in blood, although it was equivalent in both tissues and blood from benign breast tumours.
\end{abstract}

Keywords: Checkpoints, Gene Expression, CTLA-4, FOXP3, Breast Cancer (BC)

\section{Introduction}

$\mathrm{BC}$ is one of the most frequent cancers in women, and it has a high fatality rate. Breast cancer was predicted to be responsible for 29 percent of cancer diagnoses and 15 percent of cancerrelated deaths $[1,2]$. The host immune response appears to play a substantial influence in the prognosis of malignant tumours, according to accumulated data [3]. The relevance of the host immune response and immunological tolerance in the development of neoplastic illness was revealed by these findings [4]. Normally, the immune system plays a vital role in defence against malignant tumours so that the compromised patients have an increasingly significant incidence of malignancy [5]. Based on the evidence, the immune system's primary function is to recognise and kill cancerous cells as soon as they arise [6]. Unfortunately, tumour cells can evade immune detection through a variety of methods, including down-regulation of immune responses [7]. Some cancerous cells can protect themselves from killing by the immune system through stimulating immune checkpoints. Immune checkpoints are important in the self-tolerance process because they restrict the immune system from attacking cells randomly [8]. Those immunological checkpoints are currently being exploited as cancer therapeutic targets. Clinical trials using checkpoint inhibitors of solid tumours revealed a clear response that could not be replicated by other medication $[9,10]$. Cancer immunotherapy aims to improve the immune system's ability to 
recognise tumour cells by disrupting the mechanisms that tumour cells use to escape and suppress immunological responses [11]. One of the main immune regulatory molecules is Treg cells regulatory. These cells play an essential role in the regulation of immune activities and keep the effect of self-antigens. This can be achieved by different techniques, ex. suppression of antigenpresenting cells via CTLA-4 and secretion of immunomodulatory cytokines (IL-10, TGF- $\beta$, and IL-35) $[12,13]$. Forehead Box P3 (FOXP3) is a master transcription factor that controls Treg cell development, maintenance, and suppressor activity $[14,12,15]$. Treg cells are crucial immunemediated inflammation, but they've also been important mediators of active immune evasion; reducing these cells can boost endogenous anti-tumour immunity and immunotherapy efficacy. Many researchers have looked into Treg cell are immunosuppression in cancer patients [16, 17]. Treg cells suppress immune cells such as CD8+ T, NK, B cells, and APCs [18, 19]. The chemokine CCL22, which is released by tumour cells, collects them in tumour tissues $[18,19]$. Increasing the number of Treg cells promotes cancer progression by weakening the immune system and allowing tumour cells to avoid detection and elimination by the immune system. Tumor growth may be aided by suppressing cell-mediated immunity and increasing the number and/or function of Treg cells [16]. Some indicators, such as FOXP3, Cytotoxic T lymphocyte antigen-4 (CTLA-4) and glucocorticoid-induced TNFR family-related gene (GITR), alter Treg cells' immunosuppressive capabilities [20,21]. Despite the fact that numerous studies have been done on measuring FOXP3 and CTLA-4 expression in the peripheral blood of breast cancer patients [22, 23], there has been little research done on measuring FOXP3 and CTLA-4 in malignant tissues. The goal of this study was to compare and assess the expression of FOXP3 and CTLA- 4 in the tumour microenvironment and peripheral blood of breast cancer patients.

\section{Materials and Methods}

\subsection{Participants}

Between August 2020 and February 2021, a total of 51 women, aged 30 -63 years with breast cancer who attended cancers centre/AL-Sadr teaching hospital, Basra, Iraq, were involved in the present study. Permission from the doctor was acquired, and each participant signed a consent form. The patients had just been diagnosed with cancer and were included in the trial before starting chemotherapy, radiation, or immunotherapy. Expert oncologists confirm the diagnosis of breast cancer based on specific surgical and pathological findings. To validate the diagnosis, all patients completed a medical status questionnaire. The questionnaire asked about sex, age, the presence or absence of another chronic disease, family history, and drug use.

Two millilitres of venous blood samples and tissue specimens (Formalin fixation and paraffin embedding, FFPE) were collected from all participants including 36 patients (malignant cases) and 15 women with breast benign tumour as a positive control.

\subsection{Extraction and Transcription of Real-Time PCR}

PB (peripheral blood) and FFPE (Formalin-Fixed Paraffin-Embedded) specimens using the Geneaid kit, the RNA is extracted (Geneaid, Taiwan) and PROMIGA kit (PROMIGA, USA) 
based on producer's recommendations. A Nanodrop spectrophotometer was used to assess the purity and concentration of total RNA in all samples. To make cDNA from extracted RNA, a complementary DNA (cDNA) synthesis kit (OptiScriptTM RTSystem ONE-STEPRTPCRPreMix, Korea) was employed. The reverse transcription process includes adding a reverse transcription enzyme, heating it to $45^{\circ} \mathrm{C}$ for 35 minutes, and then cooling it to $95^{\circ} \mathrm{C}$ for 5 min, for reversing halt transcription enzyme from working.

The gene of FOXP3 - CTLA-4 was investigated using a real-time PCR apparatus (Applied Biosystems, USA) with 10 litres of SYBR green master mix, 2 litres of template cDNA, 1 litre of particular primers (10 pmol stock), and 6 litres of nuclease-free water. Bioneer Company synthesised the primers (Korea). Table 1 lists the primer sequences that were employed. $95^{\circ} \mathrm{C}$ for $1 \mathrm{~min}, 40$ cycles of $95^{\circ} \mathrm{C}$ for $15 \mathrm{sec}, 55-60^{\circ} \mathrm{C}$ for 1 minute, $95^{\circ} \mathrm{C}$ for 15 seconds was the thermal cycler's schedule.

The GAPDH gene was utilised as an internal control to normalise the amplified target genes. The quantity of FOXP3 and CTLA-4 in PB or FFPE expressed as a percentage of the amount of GAPDH determined using the $2^{-\Delta \Delta C T}$ algorithm.

Table 1. PCR Primers for amplification of the genes encode CTLA-4 and Foxp3.

\begin{tabular}{|c|c|c|c|}
\hline Gene & Primers & Sequence & Reference \\
\hline \multirow[t]{2}{*}{ CTLA4 } & Forward & 5-GCCCTGCACTCTCCTGTTTTT-3 & \multirow[t]{6}{*}{$\begin{array}{l}\text { (Khalife et al. } \\
\text { 2018) [24] }\end{array}$} \\
\hline & Reverse & 5-GGTTGCCGCACAGACTTCA-3 & \\
\hline \multirow[t]{2}{*}{ Foxp3 } & Forward & 5-GTGGCCCGGATGTGAGAAG-3 & \\
\hline & Reverse & 5-GGAGCCCTTGTCGGATGATG-3 & \\
\hline \multirow[t]{2}{*}{ GAPDH } & Forward & 5-TTCCAATATGATTCCACCCA-3 & \\
\hline & Reverse & 5-GATCTCGCTCCTGGAAGATG-3 & \\
\hline
\end{tabular}

\subsection{Statistical Analysis}

The data concluded the average, Std Dev., and standard error. The arithmetic comparison was done using (SPSS version 25) and the ANOVA and Mann-Whitney U tests, where needed. When $\mathrm{P}$ is less than 0.05 the data are statistically significant.

\section{Results}

The expression of CTLA-4 and FOXP3 was determined in tissues (FFPE) and peripheral blood (PB) of 36 women with $\mathrm{BC}$ and 15 positive controls (breast benign tumour) with mean age: 47 \pm 7.8 and $42 \pm 10((\mathrm{P}>0.05)$ years, respectively. The quantity of FOXP3 and CTLA-4 expression in the PB or FFPE expressed as a percentage of the amount of GAPDH expression determined using the $2^{-\Delta \Delta \mathrm{CT}}$ method. 


\subsection{The Expression of CTLA4 By Tissues and Blood}

Table 2 and Fig 1 show the fold change in CTLA-4 gene in tissues and blood of women with $\mathrm{BC}$ and a positive control group (breast benign tumour).

CTLA-4 in the tissues was substantially higher than in blood in control BC with $(\mathrm{P}<0.02)$. There was no difference in CTLA-4 mRNA in tissues or blood in the group with breast benign tumours.

CTLA-4 expression was considerably lower in blood from individuals with breast cancer compared to blood from the benign group $(\mathrm{P}<0.001)$. In addition, CTLA-4 expression in breast cancer tissues was lower than in the benign group, but the difference was not significant.

\subsection{The Expression of FOXP3 by Tissues and Blood}

The amount of the FOXP3 mRNA was in tissue from BC non-control was higher than the expression in blood but with less significant level (Fig. 2 and Table 2). FOXP3 mRNA was similar in both tissues and blood from breast benign tumours (positive control) and higher compared with breast cancer patients (Fig 1 and Table 2).

Table 2. The CTLA-4 and FOXP3 in blood and tissues from patients with BC and positive control group (Breast benign tumour).

\begin{tabular}{|c|c|c|c|}
\hline \multirow{2}{*}{$\begin{array}{c}\text { Group } \\
\text { N=36 }\end{array}$} & $\begin{array}{c}\text { Source of } \\
\text { mRNA }\end{array}$ & CTLA-4 expression & FOXP3 expression \\
\hline \begin{tabular}{c} 
Breast cancer \\
\cline { 2 - 4 }
\end{tabular} & Blood & $0.032373 \pm 0.21$ & $0.318797 \pm 0.42$ \\
\hline $\begin{array}{c}\text { Positive control } \\
\text { group } \\
\text { N=15 }\end{array}$ & Blood & $0.265997 \pm 0.27$ & $0.697323 \pm 0.26$ \\
\cline { 2 - 4 } & Tissue & $1.010447 \pm 0.28$ & $1.003004 \pm 0.47$ \\
& & & $1.000194 \pm 0.30$ \\
\hline
\end{tabular}




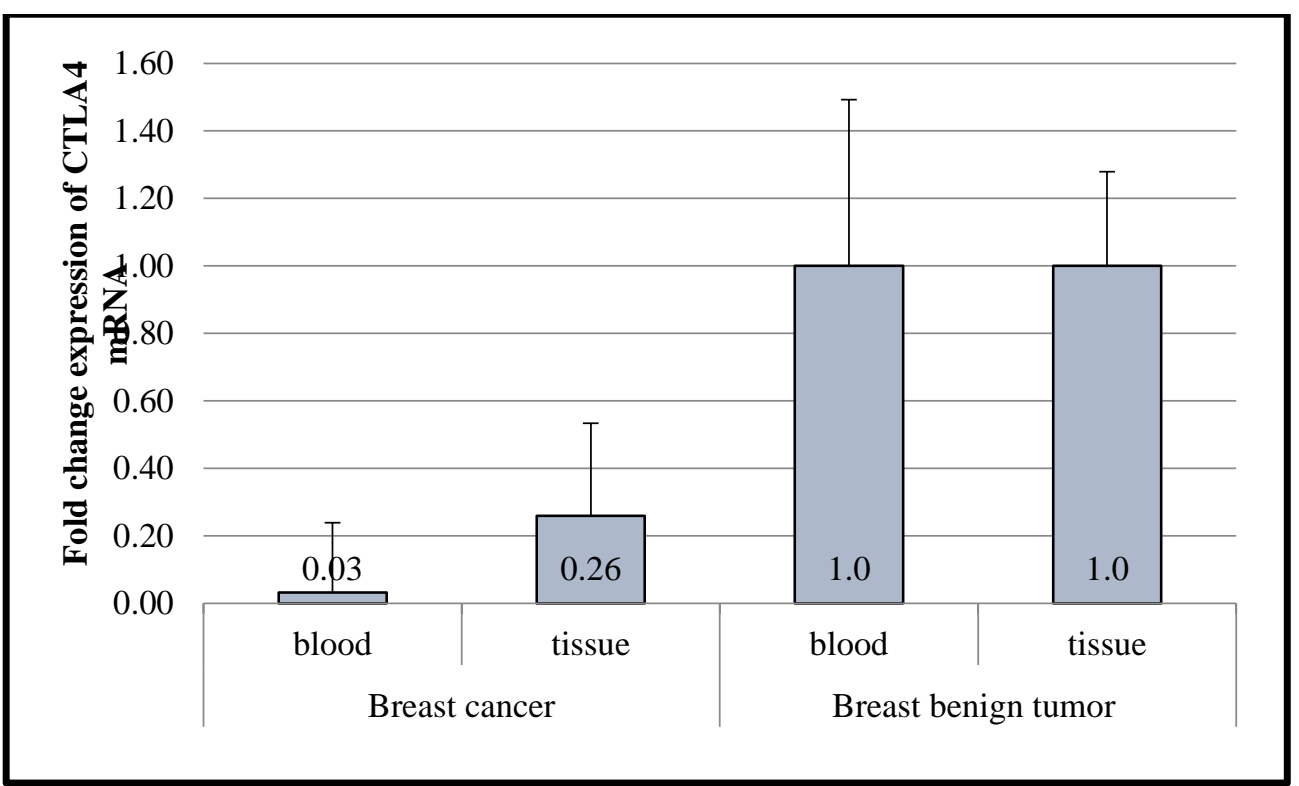

Fig 1. CTLA-4 mRNA in tissues and blood from BC non-control a positive control group (breast benign tumour). CTLA-4 in the tissues of breast cancer patients was substantially higher than in the blood.

No difference in CTLA-4 mRNA expression in tissues and blood in a breast benign tumour. CTLA-4 expression in the blood and tissues of $\mathrm{BC}$ non-control detected as less than the benign group.

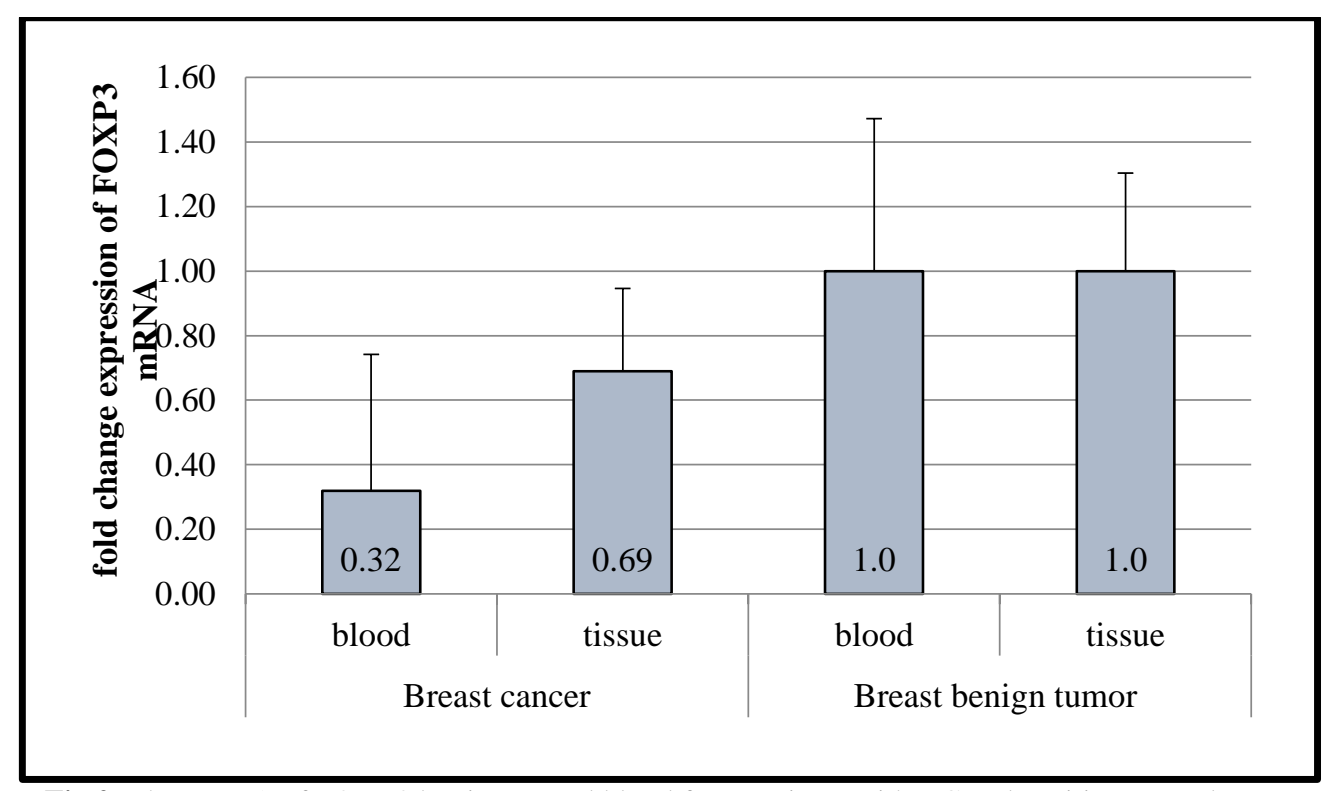

Fig 2. The mRNA of FOXP3 by tissues and blood from patients with $\mathrm{BC}$ and positive control group (breast benign tumour). The FOXP3 in tissue from BC was higher than the expression in blood, while it was similar in both tissues and blood from breast benign tumours. The expression of FOXP3 in blood and tissues from patients with $\mathrm{BC}$ was lower compared with the benign group. 


\section{Discussion}

In breast cancer, the tumour microenvironment is becoming more well recognised as a significant role in tumour growth and a possible therapeutic target. Multiple cell types are seen in both intratumor (in the breast) and distant (metastatic) tumours. An extracellular matrix, soluble components (cytokines, growth factors, hormones, and enzymes), and physical features ( $\mathrm{pH}$ and oxygen concentration) are also present [25]. soluble factors and cells of suppressive immune work synergistically to obstruct efficient antitumor immunity and promote breast cancer development and spread. Immunological suppressive systems such as immune checkpoints have evolved in the immune system to defend against autoimmunity by developing tolerance to self-antigens. Tumors use immunosuppressive pathways to dampen antitumor responses, allowing them to avoid identification and eradication by the immune system [26, 27]. The immune checkpoints that we investigated is CTLA-4 and Foxp3 were recruited by the immune system as regulatory molecules and used or induced by tumour cells to escape from immune surveillance. We compare the expression of CTLA-4 and Foxp3 in tissue microenvironment and peripheral blood. This study achieved with needs for breast biopsy as a specimen from malignant or benign tumours. Given such an invasive method a comparative study between expression of immune checkpoints in microenvironment tissue and peripheral blood was arising. According to our knowledge, such studies are rare and sparse. The study of checkpoints expression is useful not only for avoiding intrusive procedures, but also for identifying key aspects in tumour genesis and growth in terms of a metric of therapy response. Changes in epigenetic could lead to aberrant gene in tumour microenvironment cells [25], and gene expression profiles obtained from tumour stroma can predict clinical prognosis [26]. In light of these recent findings, the $\mathrm{BC}$ environment is gaining popularity and thus a possible therapeutic and new medicines could be targeted.

The present study showed that CTLA-4 gene expression in breast cancer tissues was significantly higher than peripheral blood and lower than the expression in tissues and blood from the control group (benign tumour). These discoveries will aid in early detection, and the development of therapeutic methods for BC. However, tissue biopsy for breast cancer tissues is still required. Using the ELISA approach, Isitmangil $\mathrm{G}$ et al. [28] got the serum level of CTLA4 higher in non-control than in controls. CTLA-4 expression was shown to be high in tumour cells but very mildly positive or negative in normal breast tissue by Moa et al [29].

FOXP3 was the other immune checkpoint studied here. Its sign in in breast cancer tissues is expected higher in blood but it is not at the significant level, which could be owing to the limited sample size. Foxp3 expression in tumours, on the other hand, was linked to a lower overall survival rate, and risk rose as Foxp3 expression increased. Foxp3 was also a good predictor but not as reappearance risk.

Finally, we hope that our findings will be useful in early detection of BC.

\section{Acknowledgments}

Authors thank Ms. Zahraa H Abdulkareem for her excellent technical help.

\section{References}


1. Mattiuzzi, C., \& Lippi, G. Current cancer epidemiology. Journal of epidemiology and global health. 2019; 9(4), 217.

2. Siegel, R. L., Miller, K. D., \& Jemal, A. Cancer statistics, 2016. CA: a cancer journal for clinicians. 2016; 66(1), 7-30.

3. Chen, D. S., \& Mellman, I. Oncology meets immunology: the cancer-immunity cycle. Immunity. 2013; 39(1), 1-10.

4. Galon, J., Mlecnik, B., Bindea, G., Angell, H. K., Berger, A., Lagorce, C., ... \& Pagès, F. Towards the introduction of the 'Immunoscore'in the classification of malignant tumours. The Journal of pathology. 2014; 232(2), 199-209

5. Casey, S. C., Li, Y., \& Felsher, D. W. An essential role for the immune system in the mechanism of tumor regression following targeted oncogene inactivation. Immunologic research. 2014; 58(2-3), $282-291$.

6. Monzavi-Karbassi, B., Pashov, A., \& Kieber-Emmons, T. Tumor-associated glycans and immune surveillance. Vaccines. 2013; 1(2), 174-203.

7. Spranger, S. Mechanisms of tumor escape in the context of the T-cell-inflamed and the non-T-cellinflamed tumor microenvironment. International immunology. 2016; 28(8), 383-391.

8. Pardoll, D. M. The blockade of immune checkpoints in cancer immunotherapy. Nature Reviews Cancer. 2012; 12(4), 252-264.

9. Tanday, S. Nivolumab for recurrent or metastatic head and neck cancer. The Lancet Oncology. 2016; 17(11), e483.

10. Wang, X., Bao, Z., Zhang, X., Li, F., Lai, T., Cao, C., ... \& Ying, S. Effectiveness and safety of PD1/PD-L1 inhibitors in the treatment of solid tumors: a systematic review and metaanalysis. Oncotarget. 2017; 8(35), 59901.

11. Sheikhi, A., Jafarzadeh, A., Kokhaei, P., \& Hojjat-Farsangi, M. Whole tumor cell vaccine adjuvants: comparing IL-12 to IL-2 and IL-15. Iranian Journal of Immunology. 2016; 148-166.

12. Jafarzadeh, A., Jamali, M., Mahdavi, R., Ebrahimi, H. A., Hajghani, H., Khosravimashizi, A., ... \& Daneshvar, H. Circulating levels of interleukin-35 in patients with multiple sclerosis: evaluation of the influences of FOXP3 gene polymorphism and treatment program. Journal of Molecular Neuroscience. 2015c; 55(4), 891-897.

13. Takeuchi, Y., \& Nishikawa, H. Roles of regulatory $\mathrm{T}$ cells in cancer immunity. International immunology. 2016; 28(8), 401-409.

14. Etesam, Z., Nemati, M., Ebrahimizadeh, M. A., Ebrahimi, H. A., Hajghani, H., Khalili, T., ... \& Jafarzadeh, A. Altered expression of specific transcription factors of Th17 (ROR $\gamma \mathrm{t}, \mathrm{ROR} \alpha)$ and Treg lymphocytes (FOXP3) by peripheral blood mononuclear cells from patients with multiple sclerosis. Journal of Molecular Neuroscience. 2016; 60(1), 94-101.

15. Szylberg, Ł., Karbownik, D., \& Marszałek, A. The role of FOXP3 in human cancers. Anticancer research. 2016; 36(8), 3789-3794.

16. Hatanaka, H., Ishizawa, H., Nakamura, Y., Tadokoro, H., Tanaka, S., Onda, K., ... \& Hirano, T. Effects of vitamin $\mathrm{K} 3$ and $\mathrm{K} 5$ on proliferation, cytokine production, and regulatory $\mathrm{T}$ cell-frequency in human peripheral-blood mononuclear cells. Life science. 2014; 99(1-2), 61-68.

17. Tohyama, N., Tanaka, S., Onda, K., Sugiyama, K., \& Hirano, T. Influence of anticancer agents on cell survival, proliferation, and CD4+ CD25+ Foxp3+ regulatory $\mathrm{T}$ cell-frequency in human peripheral-blood mononuclear cells activated by $\mathrm{T}$ cell-mitogen. International immunopharmacology. 2013; 15(1), 160-166.

18. Jafarzadeh, A., Fooladseresht, H., Minaee, K., Bazrafshani, M. R., Khosravimashizi, A., Nemati, M., ... \& Ghaderi, A. Higher circulating levels of chemokine CCL22 in patients with breast cancer: evaluation of the influences of tumor stage and chemokine gene polymorphism. Tumor Biology. 2015a; 36(2), 1163-1171.

19. Muenst, S., Läubli, H., Soysal, S. D., Zippelius, A., Tzankov, A., \& Hoeller, S. The immune system and cancer evasion strategies: therapeutic concepts. Journal of internal medicine. 2016; 279(6), 541562.

20. Nasser, A., Khosravi, A., \& Azizian, R. The role of T regulatory lymphocytes in lymphoma. Middle East Journal of Cancer. 2014; 5(2), 55-66. 
21. Rathod, S. B., Das, R., Thanapati, S., Arankalle, V. A., \& Tripathy, A. S. Suppressive activity and altered conventional phenotype markers/mediators of regulatory $\mathrm{T}$ cells in patients with self-limiting hepatitis E. Journal of Viral Hepatitis; 2014; 21(2), 141-151.

22. Jaberipour, M., Habibagahi, M., Hosseini, A., Habibabad, S. R., Talei, A., \& Ghaderi, A. Increased CTLA-4 and FOXP3 transcripts in peripheral blood mononuclear cells of patients with breast cancer. Pathology \& Oncology Research. 2010; 16(4), 547-551.

23. Kawaguchi, K., Suzuki, E., Yamaguchi, A., Yamamoto, M., Morita, S., \& Toi, M. Altered expression of major immune regulatory molecules in peripheral blood immune cells associated with breast cancer. Breast Cancer. 2017; 24(1), 111-120.

24. Khalife, E., Khodadadi, A., Talaeizadeh, A., Rahimian, L., Nemati, M., \& Jafarzadeh, A. Overexpression of regulatory T cell-related markers (FOXP3, CTLA-4 and GITR) by peripheral blood mononuclear cells from patients with breast cancer. Asian Pacific journal of cancer prevention: APJCP. 2018; 19(11), 3019.

25. Coleman RE, Gregory W, Marshall H, Wilson C, Holen I: The metastatic microenvironment of breast cancer: clinical implications. Breast 2013; 22(suppl 2): S50-S56.

26. 26- Folgueira MA, Maistro S, Katayama ML, Roela RA, Mundim FG, Nanogaki S, de Bock GH, Brentani MM: Markers of breast cancer stromal fibroblasts in the primary tumour site associated with lymph node metastasis: a systematic review including our case series. Biosci Rep 2013; 33: e00085.

27. Finak G, Bertos N, Pepin F, Sadekova S, Souleimanova M, Zhao H, Chen H, Omeroglu G, Meterissian S, Omeroglu A, Hallett M, Park M: Stromal gene expression predicts clinical outcome in breast cancer. Nat Med 2008; 14: 518-527.

28. Isitmangil G, Gurleyik G, Aker FV, Coskun C, Kucukhuseyin O, Arikan S, Turan S, Talu CK, Dogan MB, Farooqi AA, Yaylim I. Association of CTLA4 and CD28 Gene Variants and Circulating Levels of Their Proteins in Patients with Breast Cancer. In Vivo. 2016 Jul-Aug;30(4):485-93.

29. Mao H, Zhang L, Yang Y, Zuo W, Bi Y, Gao W, Deng B, Sun J, Shao Q, Qu X. New insights of CTLA-4 into its biological function in breast cancer. Curr Cancer Drug Targets. 2010 Nov;10(7):72836. 